\title{
Abrupt Rise of the Longitudinal Recoil Ion Momentum Distribution for Ionizing Collisions
}

\author{
Th. Weber, ${ }^{1, *}$ Kh. Khayyat, ${ }^{1,2}$ R. Dörner, ${ }^{3}$ V. D. Rodríguez, ${ }^{4}$ V. Mergel, ${ }^{1}$ O. Jagutzki, ${ }^{1}$ L. Schmidt, ${ }^{1}$ \\ K. A. Müller, ${ }^{1}$ F. Afaneh, ${ }^{1}$ A. Gonzalez, ${ }^{5}$ and H. Schmidt-Böcking ${ }^{1}$ \\ ${ }^{1}$ Institut für Kernphysik, August-Euler-Straße 6, 60486 Frankfurt am Main, Germany \\ ${ }^{2}$ Department of Physics, University of Missouri, Rolla, Missouri \\ ${ }^{3}$ Fakultät für Physik, Universität Freiburg, Germany \\ ${ }^{4}$ Departamento de Física, FCEN, Universidad de Buenos Aires, Buenos Aires, Argentina \\ ${ }^{5}$ Centro Atomico Bariloche, $8400 \mathrm{~S} C$ de Bariloche, Argentina
}

(Received 8 June 2000)

\begin{abstract}
We report on the experimental observation of an abrupt rise in the longitudinal momentum distribution of recoil ions created in proton helium collision. The details of this structure can be related to electrons traveling with the velocity of the projectile [electron capture to the continuum (ECC)]. The longitudinal as well as the transverse distribution of the recoil ions can be explained as a continuation of the momentum distribution from ions resulting from electron capture illustrating the smooth transition from the capture to bound states of the projectile to the ECC.
\end{abstract}

DOI: $10.1103 /$ PhysRevLett.86.224

PACS numbers: $34.50 . \mathrm{Bw}, 34.70 .+\mathrm{e}$

The single ionization of an atom by a charged particle represents an excellent example for a nonstationary quantum mechanical three-body problem, which even 74 years after the formulation of the Schrödinger equation remains a major challenge for theory (see [1]). The momentum distribution of the fragments in the final state of an atomic collision is the main source of information on the dynamics of such ionization processes. Of particular interest are discrete structures in these momentum distributions, which are directly linked to a particular mechanism or to a special kinematical scenario of how the ionization proceeds. In the momentum distribution of ionized continuum electrons three of such distinct structures are known: (1) A spherical ridge in the velocity space, centered on the projectile velocity $v_{p}$, resulting from the binary encounter between the projectile and a quasifree target electron. And (2) and (3) two peaks at the target and at the projectile velocity, resulting from very slow electrons ["target cusp" or electron capture to the target (ECT), see $[2,3]]$ and electrons traveling with the projectile without being bound [electron capture to the continuum (ECC); see [4-6] and references therein, as well as [7] for a review]. For the ECC processes the conspicuous cusp shaped double differential cross sections were assumed to be due to the long-range nature of the Coulomb interaction of the electron and the charged projectile in the final state. But contrary to the prominently structured electron momentum distribution, all experimental momentum distributions of the recoiling ion in ionizing collisions are remarkably smooth and without any structures (see, e.g., [8-11]).

In this Letter we report on the experimental observation of a new discrete structure in the $\mathrm{He}^{1+}$ recoil-ion longitudinal momentum $p_{z r}$ distribution for the reaction

$$
d^{+}+\mathrm{He} \rightarrow d^{+}+\mathrm{He}^{1+}+e^{-} .
$$

We observe an abrupt rise in the longitudinal recoil-ion momentum distribution that is related to the ECC processes and which for the first time confirms a theoretical prediction of Rodríguez et al. [12].

The conservation laws of energy and momentum connect the final state momenta of the three particles on the right-hand side of Eq. (1). They restrict the number of linearly independent observables (neglecting the spin) to $9-4=5$. For heavy projectile impact one can easily show (see [13]) that the recoil-ion longitudinal momentum in the final state is given by [we use atomic units (a.u.) throughout this paper]

$$
\begin{aligned}
p_{z r}^{\text {ionization }} & =\frac{E_{e}-E_{\mathrm{bind}}}{v_{p}}-\sqrt{2 E_{e}} \cos \vartheta_{e} \\
& =\frac{E_{e}-E_{\mathrm{bind}}}{v_{p}}-p_{z e},
\end{aligned}
$$

where $E_{\text {bind }}$ is the binding energy (negative value) of the electron in the initial state, $E_{e}$ is the kinetic energy of the electrons in the final state, and $\vartheta_{e}$ is the polar emission angle of the electron (in the laboratory frame). Note that there is no one to one relation between the electron energy in the laboratory frame or the projectile energy loss and the longitudinal recoil-ion momentum (see [14]). A strikingly simple interpretation of $p_{z r}^{\text {ionization }}$ is gained by transforming the electron momenta from the laboratory to the projectile rest frame (we use $*$ to indicate terms in the rest frame of the projectile). From Eq. (2) it is straightforward to derive the following result:

$$
p_{z r}^{\text {ionization }}=-\frac{v_{p}}{2}-\frac{E_{\mathrm{bind}}}{v_{p}}+\frac{E_{e}^{*}}{v_{p}}
$$

Here $E_{e}^{*}$ is the kinetic energy of the active electron seen from the moving projectile frame. For the ionization process it is clear that $E_{e}^{*}>0$ and therefore the minimum possible recoil-ion longitudinal momentum is obtained, when 
$E_{e}^{*}$ equals zero, given by

$$
p_{z r}^{\min }=-\frac{v_{p}}{2}-\frac{E_{\text {bind }}}{v_{p}} .
$$

$p_{z r}^{\min }$ corresponds to the electron emission of the ECC, i.e., electrons with no kinetic energy and velocity in respect to the moving projectile.

Equation (3) links the capture processes and the ionizing collisions. In fact, for $E_{e}^{*}<0$ the electron is in a bound state of the projectile, describing a capture reaction $d^{+}+\mathrm{He} \rightarrow d^{0}+\mathrm{He}^{1+}$ [including excited final states of the target atom; see inset of Fig. 1(a)]. In this case obviously $E_{e}^{*}$ as well as $p_{z r}^{\text {capture }}$ are quantized. Equation (3) has been widely used for high-precision energy gain spectroscopy (see [15] and references in [13]).

Details on the experimental technique of cold target recoil ion momentum spectroscopy (COLTRIMS) used for this experiment can be found elsewhere (see [8] for more detailed information on the experimental setup used for this experiment and [13] for a review). In brief, a precooled supersonic gas jet is crossed with a pulsed ion beam. Upstream and downstream of the target gas jet the ion beam is charge state analyzed by electrostatic deflectors. If a projectile is neutralized it is detected by a channel plate detector. An electrostatic field guides the recoil ions created in the overlap region onto another position-sensitive channel plate detector. From the position of impact on the detector and the time-of-flight the three components of the recoil-ion momentum vector can be determined. With this configuration we were able to measure the recoil-ion momentum distributions for all recoil ions and simultaneously the ions from single capture reaction only. Since capture leads to clearly separable lines in the recoil-ion momentum distributions [see Eq. (3) and Fig. 1], the two measured distributions can be normalized very exactly with respect to each other and the momentum distributions for the pure ionization channel can be obtained just by subtracting these two distributions. The low extraction fields and the electrostatic spatial focusing resulted in a very high resolution of \pm 0.025 a.u. in the longitudinal recoil-ion momentum crucial for this experiment [the good resolution is most obvious in the width of the capture lines in Fig. 1(c), for example]. Therefore the relative energy gain resolution of the projectile is better than $\Delta E / E<10^{-5}$. The resolution in the time-of-flight direction was \pm 0.012 a.u.

In Fig. 1 the recoil-ion longitudinal momentum distribution for the ionizing (solid line) and the capture processes (dotted line) for projectile energies of 100, 150, and $200 \mathrm{keV} / \mathrm{u}$ are shown. The very high momentum resolution achieved here is necessary to visualize and distinguish between all these structures and processes. At all three velocities an abrupt rise of the $p_{z r}$ distribution for the ionization is found. The existence of a minimum value of $p_{z r}^{\min }$ [indicated by ECC in Fig. 1(b)] is just a consequence of momentum and energy conservation, i.e., purely a consequence of kinematics. It bears no information on

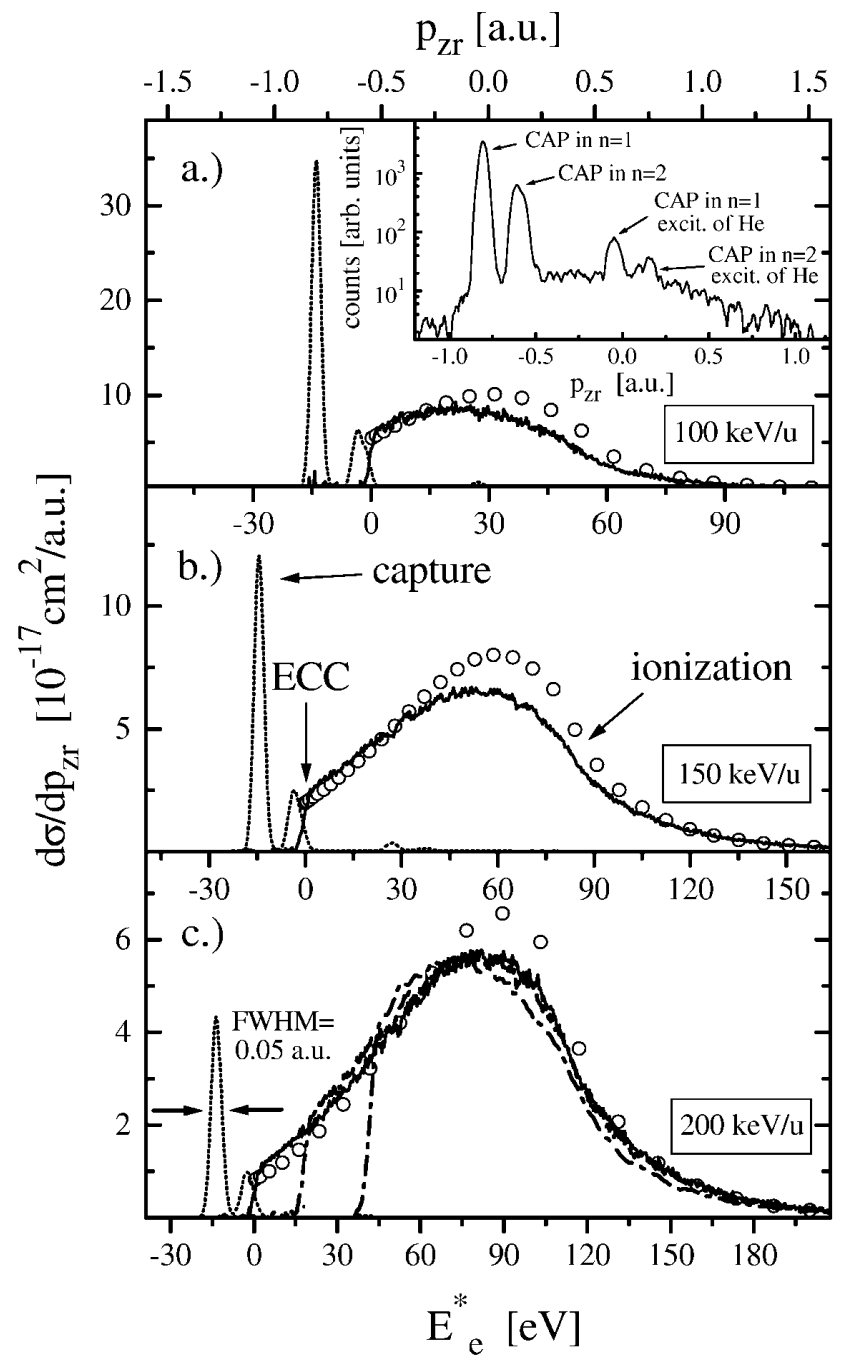

FIG. 1. Longitudinal recoil-ion momentum (top-scale) distribution for three different kinetic energies of the projectile (100, $150,200 \mathrm{keV} / \mathrm{u}$ ). The solid lines indicate the ionization processes, the dotted curves show the different capture channels, and the open circles are results from CDW-EIS theory. A logarithmic plot of the recoil-ion momentum distribution of the capture processes can be seen as an inset of (a). Electron capture in $n=1, n=2$ of the projectile as well as the same capture channels with simultaneous excitation of the helium target are visible. The scale at the bottom of each spectrum indicates either the kinetic energy of the electrons in the projectile frame $E_{e}^{*} \geq 0$ or the bound electron capture energy for $E_{e}^{*}<0$. In (c) an overlay of all ionization distributions are shown (dash-dotted lines) which are normalized to the maximum of the cross section for $200 \mathrm{keV} / \mathrm{u}$.

the dynamics or any other detail of the reaction. For example, this would also occur for antiproton or even any neutral particle impact as well [14]. The physical information lies in the fact of which way the kinematically allowed phase space is populated. It is remarkable that the cross section $d \sigma / d p_{z r}$ has a finite value at $p_{z r}^{\min }$, i.e., an abrupt rather than a smooth rise. Rodríguez and co-workers have predicted this abrupt rise within a continuum distorted wave eikonal initial state (CDW-EIS) calculation 
(see [12]). They argued that this is a mirror image of the ECC peak in the electron momentum distribution. If one assumes a homogeneous population (i.e., no cusplike structure) of the electron momentum phase space in the vicinity of the ECC (i.e., $d^{3} \sigma / d p_{e x} d p_{e y} d p_{e z}=$ const), this would result in a smooth rise like $d \sigma / d p_{r}=\sqrt{p_{r}-p_{r}^{\min }}$. An abrupt rise requires $d^{3} \sigma / d p_{e x} d p_{e y} d p_{e z}$ diverging exactly like $1 / \sqrt{p_{e x}^{2}+p_{e y}^{2}+\left(p_{e z}-v_{p}\right)^{2}}$ (see [12,14]). The present results should be considered as a sensitive verification of the actual ECC cusp structure.

Using Eq. (3) one can also interpret the $p_{z r}$ distribution of Fig. 1 as a cross section single differential in the electron energy $\left(E_{e}^{*}\right)$ seen from the moving projectile. These cross sections are simply related by $v_{p}\left(d \sigma / d E_{e}^{*}\right)=d \sigma / d p_{z r}$. $E_{e}^{*}$ is indicated by the additional bottom scales in Fig. 1. In the electron spectra of the laboratory frame the ECC leads to a divergence in the doubly differential cross section $d^{2} \sigma /\left(d \Omega d E_{e}\right)$. In the projectile frame this divergence is not present, and $d \sigma / d E_{e}^{*}$ shows a smooth transition across the continuum threshold (see Fig. 1). Thus the capture to highly excited states joins the ionization continuum continuously across the ECC edge. Our data have been normalized to the total cross sections from [16]. CDW-EIS calculations for the ionization channel reproduce the general feature and the abrupt rise (see circles in Fig. 1). The discrepancy in the details of the shape of the distribution, however, indicates the problems of perturbation theory at these relative low velocities $v_{p}$.

In addition to the longitudinal momentum distribution for $200 \mathrm{keV} / \mathrm{u}$ in Fig. 1(c) the momentum distributions for the ionization processes of Figs. 1(a) and 1(b) are overlaid (dash-dotted lines). They have an almost velocity independent shape but are cut at different longitudinal momentum due to the different projectile velocities. This also shows the physical reason for the rapid change in the ratio of capture to ionization. It is the kinematics that moves the ion momenta related to capture into a region of smaller population decreasing this proportion. At the same time the cutoff in the ionization continuum also moves backwards. The overall shape is very similar to the Compton profile (see also [9]) suggesting that this distribution is highly influenced by the initial state. In the initial state of an atom a backward momentum of the nucleus higher than the cutoff momentum corresponds to an electron moving forward faster than the projectile. Thus the projectile is unable to transfer momentum to the target electron and ionize a helium atom with such initial state momentum via pure electron-projectile interaction resulting in some sort of sound barrier in the longitudinal recoil-ion momentum distribution moving backward with the projectile velocity.

Additional information can be gained from the doubly differential cross section $d^{2} \sigma /\left(d p_{z r} d p_{t r}\right)$ (see Fig. 2). The transverse recoil-ion momentum $p_{t r}$ for the capture reaction is simply the mirror image of the projectile scattering. For the ionization it is the sum of the projectile and the electron transverse momentum. Thus it is indirectly linked

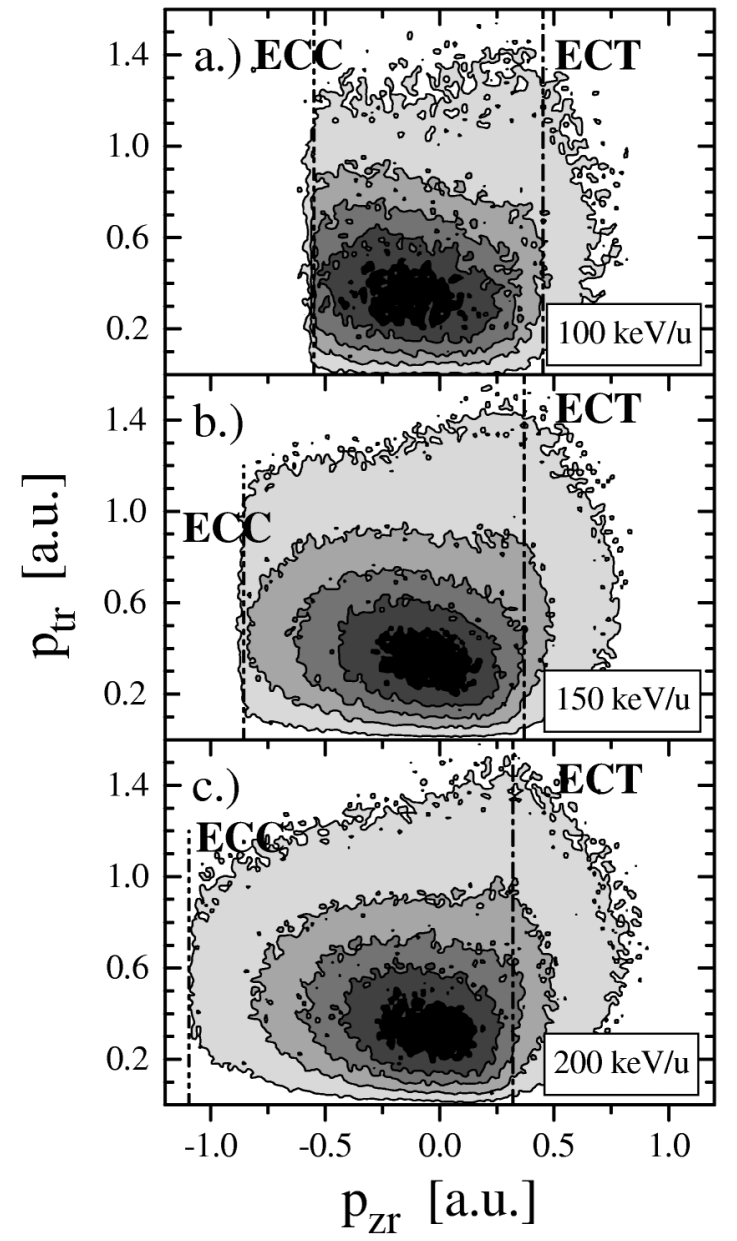

FIG. 2. The momentum distribution for the recoil ions of the ionization process for three different kinetic energies of the projectile. The abscissa shows the momentum in the longitudinal direction and the ordinate represents the transverse momentum. The doubly differential cross section $d^{2} \sigma /\left(d p_{z r} d p_{t r}\right)$ is plotted in linear scale. The dash-dot-dotted lines (left-hand side) indicate the electron capture to the continuum (ECC) while the dash-dotted lines (right-hand side) indicate the target cusp (ECT).

to the impact parameter as well as to the electron emission dynamics [9]. At least two trends can be observed in the cross section $d^{2} \sigma /\left(d p_{z r} d p_{t r}\right)$ : First, the production of ions with $p_{z r}$ close to $p_{z r}^{\min }$ and thus the production of high energy electrons in the forward direction close to the ECC peak leads to larger transverse recoil-ion momenta than the ions with more longitudinal momentum in the forward direction. The dominant contribution to the recoil-ion doubly differential cross section $d^{2} \sigma /\left(d p_{z r} d p_{t r}\right)$ peaks at small momentum and can be associated with low energetic electron emission.

The second effect is the shoulder/convexity moving with the kinetic energy of the projectile in the double differential cross section $d^{2} \sigma /\left(d p_{z r} d p_{t r}\right)$ indicated by the dash-dotted lines (ECT) at the right-hand side of the spectra in Fig. 2 (the dash-dot-dotted lines at the left-hand side indicate the ECC processes). This ECT line at $-E_{\text {bind }} / v_{p}$ shows the 


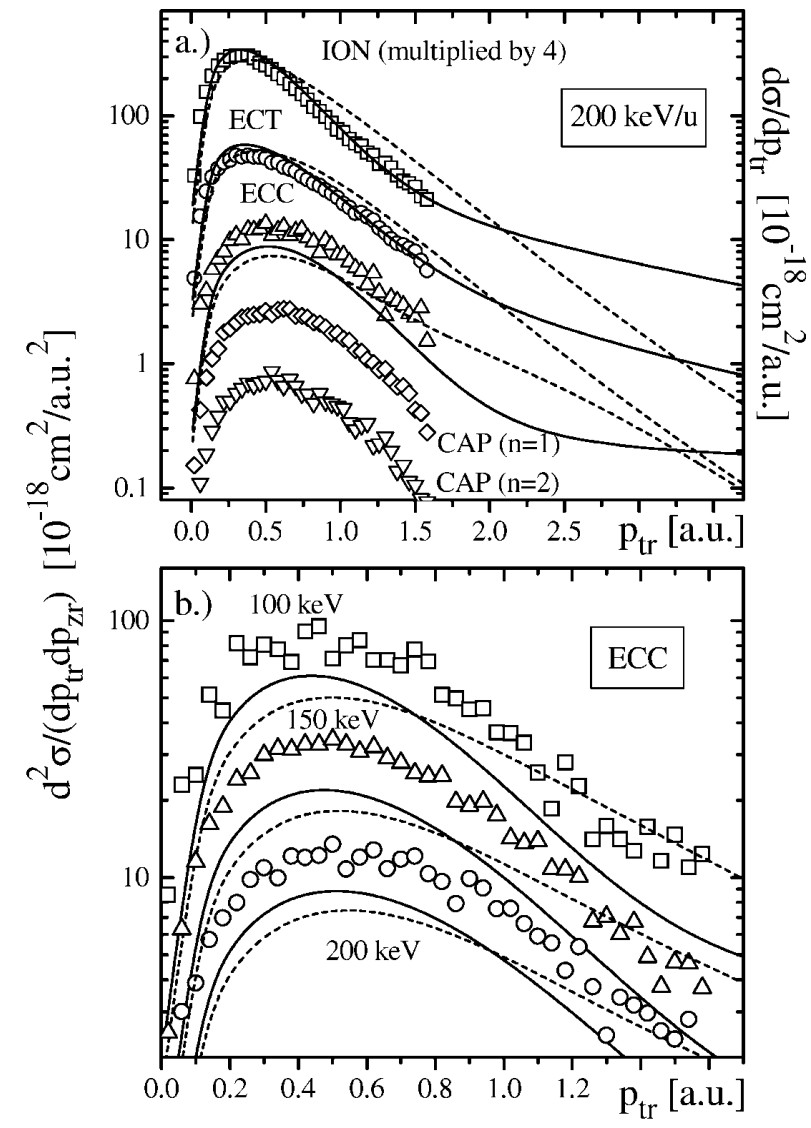

FIG. 3. The transverse recoil-ion momentum distributions for different processes and different intervals of the longitudinal recoil-ion momentum. (a) Squares (ION): Plain ionization $\left(-0.13<p_{z r}<0.08\right.$ a.u.). Circles (ECT): Target cusp $\left(0.35<p_{z r}<0.45\right.$ a.u.). Up triangles (ECC): Cusp $\left(-0.86<p_{z r}<-0.78\right.$ a.u.). Diamonds (CAP): Capture in $n=1$. Down triangles (CAP): Capture in $n=2$. The ordinate represents the cross section doubly differential in $p_{z r}$ and $p_{t r}$ for ION and ECT as well as for ECC. The $y$ axis is single differential in $p_{t r}$ for capture (CAP). The solid lines are results from CDW-EIS theory including the interaction between all three particles; the dashed lines show CDW-EIS calculation in which the nuclear-nuclear repulsion has been omitted. The kinetic energy of the projectile is $200 \mathrm{keV} / \mathrm{u}$. (b) ECC processes for three different kinetic energies of the projectile.

locus of events with zero energy electrons [see Eq. (2)]. Schmitt et al. have recently reported on the cusplike structure (called target cusp) in the electron space at momentum zero $\left(E_{e}=0\right)$ (see [2] and also [3]).

The transverse momentum transfer to the recoil-ion as well as the projectile scattering is the result of a subtle interplay in the three-particle interaction. Here theory can help to uncover some of the underlying mechanisms. Therefore, we show in Figs. 3(a) and 3(b) the experimental transverse momentum distribution for different intervals of the longitudinal momentum in comparison with the result of the full CDW-EIS calculation (solid line) and with the CDW-EIS calculation where the repulsion between the two nuclei has been switched off (dashed line). The full calculation shows a narrower ion momentum distribution, which is in much better agreement with all experimental data than the model without the nuclear repulsion. This indicates that the nuclear momentum exchange counteracts the momentum transfer between the electron and the target nucleus, resulting in a smaller mean transverse momentum of the recoil ion. Large transverse recoil-ion momenta, however, are still a result of pure nuclear-nuclear scattering. It can be clearly seen that the transverse momentum distribution of the capture and the ECC channel are very similar, again highlighting the smooth transition from capture to ECC. The main contribution to the ionization channel (ION), however, peaks at smaller transverse momenta than the ECC and the capture processes.

In conclusion, we have found an abrupt rise of the recoil-ion longitudinal momentum distribution linked to the ECC electrons. At very small scattering angles $\left(<10^{-4} \mathrm{rad}\right)$ capture to projectile states of $n \geq 2$ and ionizing collision leading to the ECC show the same cross section per ion momentum phase space and the same transverse momentum exchange highlighting a smooth transition from capture to ionization in ion atom collisions.

Th. W. is grateful for the financial support of the Graduiertenförderung des Landes Hessen. R. D. acknowledges support through the Heisenberg Program of the DFG and the Adolf-Messer-Stiftung. We thank RoentDek Handels $\mathrm{GmbH}$ for the preparation of the positionsensitive detectors and other technical equipment. We thank the staff of the IKF for their excellent work at the beam line and the preparation of the ion beam. We also thank our friends and colleagues J. Ullrich, R. Moshammer, C. L. Cocke, and R. E. Olson for continuous collaboration and helpful discussions. This work is supported by the DFG, the GSI, the DAAD, and the BMBF.

*Email address: weber@hsb.uni-frankfurt.de

[1] T. N. Rescigno et al., Science 286, 2474-2479 (1999).

[2] W. Schmitt et al., Phys. Rev. Lett. 81, 4337 (1998).

[3] P. D. Fainstein, Phys. Rev. A 60, R741-744 (1999).

[4] G. B. Crooks et al., Phys. Rev. Lett. 25, 1599 (1970).

[5] W. Meckbach et al., Phys. Rev. A 24, 1793 (1981).

[6] J. Macek et al., Phys. Rev. Lett. 46, 1571 (1981).

[7] N. Stolterfoht et al., Electron Emission in Heavy Ion-Atom Collisions (Springer-Verlag, Berlin, Heidelberg, 1997).

[8] Th. Weber et al., J. Phys. B 33, 3331 (2000).

[9] R. Dörner et al., J. Phys. B 28, 435 (1995).

[10] M. Unverzagt et al., Phys. Rev. Lett. 76, 1043 (1996).

[11] P. Jardin et al., Nucl. Instrum. Methods Phys. Res., Sect. B 107, 41 (1996).

[12] V. D. Rodríguez et al., Phys. Rev. A 52, R9 (1995).

[13] R. Dörner et al., Phys. Rep. 330, 95-192 (2000).

[14] V. D. Rodríguez et al., Phys. Rev. A 53, 3335 (1996).

[15] R. Ali et al., Phys. Rev. Lett. 69, 2491 (1992).

[16] M.D. Shah et al., J. Phys. B 18, 899 (1985). 